\title{
A study of normal eyelashes in Japanese individuals
}

\author{
Mamoru Kikuchi $^{1}$, Ken Matsuda ${ }^{2}$, Yasuhiro Ishihara ${ }^{1}$, TetsuYanai ${ }^{1}$, Kiyoto Yasuta ${ }^{1}$, Ko Hosokawa $^{3}$ and Tetsuji Uemura ${ }^{1}$ \\ ${ }^{1}$ Department of Plastic and Reconstructive Surgery, Saga University Hospital, Saga, Japan \\ ${ }^{2}$ Division of Plastic and Reconstructive Surgery, Niigata University Graduate School of Medicine, Niigata, Japan \\ ${ }^{3}$ Department of Plastic and Reconstructive Surgery, Osaka University Graduate School of Medicine, Osaka, Japan
}

\begin{abstract}
Eyelashes can greatly influence the overall impression of facial features. However few detailed examinations of eyelashes in Japanese individuals have been published. The aim of the present study is clarify the characteristic of eyelashes of Japanese individuals mainly in their 20s. Data were collected from 50 healthy volunteers with no eyelash abnormalities. The length, density, form, and angle of the eyelashes were examined. Mean eyelash lengths in males and females were $7.33 \pm 0.83 \mathrm{~mm}$ and $7.47 \pm 0.68 \mathrm{~mm}$, respectively, for the upper eyelids, and $4.98 \pm 0.75 \mathrm{~mm}$ and $5.56 \pm 0.60 \mathrm{~mm}$, respectively, for the lower eyelids. Eyelash density showed a mean of $12.12 \pm 2.04$ and $15.24 \pm 4.18$ for the upper eyelids, and $5.80 \pm 1.76$ and $6.24 \pm 1.16$ for the lower eyelids. With regard to the form of the eyelashes on the upper eyelids, 10 (40\%) were curly and $15(60 \%)$ were straight in males, whereas $19(76 \%)$ were curly and $6(24 \%)$ were straight in females. All lower eyelid eyelashes were curly. Mean angles of the eyelashes in males and females were $61.4^{\circ} \pm 13.7^{\circ}$ and $71.8^{\circ} \pm 13.5^{\circ}$ for the upper eyelids, and $90.0^{\circ} \pm 10.1^{\circ}$ and $99.8^{\circ} \pm 10.8^{\circ}$ for the lower eyelids. Mean values obtained from the this study could be used as baseline values for eyelash reconstruction or treatment for eyelash hypotrichosis, and pertaining to eyelash morphology represent one of a few assessments of eyelash characteristics in Japanese adults of both sexes.
\end{abstract}

\section{Introduction}

Eyelashes can greatly affect the overall impression of facial features, and especially for women, represent something that can be enhanced by makeup to alter one's features a fair bit. Eyelashes are found on both upper and lower eyelids, but the length and density of these vary widely by ethnicity. Ethnic and individual variation in the density and length of eyelashes can also greatly influence one's appearance [1,2]. In recent years, drugs to treat eyelash hypotrichosis, such as Bimatoprost, have been marketed and received much attention [3-5]. In general, East Asian (including Japanese) individuals tend to have shorter eyelashes of a lower density relative to those in Caucasian or West Asian individuals, but few detailed examinations of eyelashes in Asian individuals have been published. The present study targeted Japanese individuals primarily in their 20s, and measured the form, length, and angle of normal eyelashes. In addition, we used a questionnaire to survey how these individuals perceive their own eyelashes.

\section{Subjects and methods}

\section{Subjects}

Fifty healthy Japanese volunteers ( 25 male, 25 female) aged between 22 and 38 years (mean, $25.0 \pm 3.6$ years) with no eyelash abnormalities were enrolled in this study. Data were collected from eyelashes of each individual's right eyelid. Individuals with histories of disease or injury to their eyelashes, as well as those who underwent plastic or ophthalmologic surgery were excluded. Data were collected from subjects who had not used mascara, eyeliner, or any other makeup from the time they woke up.

\section{Measurements}

We examined the length, density, form, and angle of the eyelashes. Eyelash length on the upper and lower eyelids was measured by the longest one in the central $2 \mathrm{~mm}$ between the medial and lateral cantus using a caliper capable of measuring in $0.25 \mathrm{~mm}$ increments. Eyelash density was evaluated by taking a scaled image with a digital camera, whereby the number of eyelashes growing in the central $2 \mathrm{~mm}$ of the upper and lower eyelids was recorded (Figures 1a and 1b). Each subject placed their forehead and chin on the device, and was photographed while maintaining a constant position across from the camera, which was fixed in place. With regard to the form (straight versus curly) and angle of the eyelashes, we digitally photographed subjects from lateral views using the same device while they maintained a relaxed primary position of the eye, with their eyes open. The angle was defined as the angle between the root of the eyelashes and the vertical line shown in Figure 2. In addition, all subjects filled out a questionnaire and evaluated their self-image with regard to their eyelashes (short, regular, long; thin, normal, thick) on a 3-point scale.

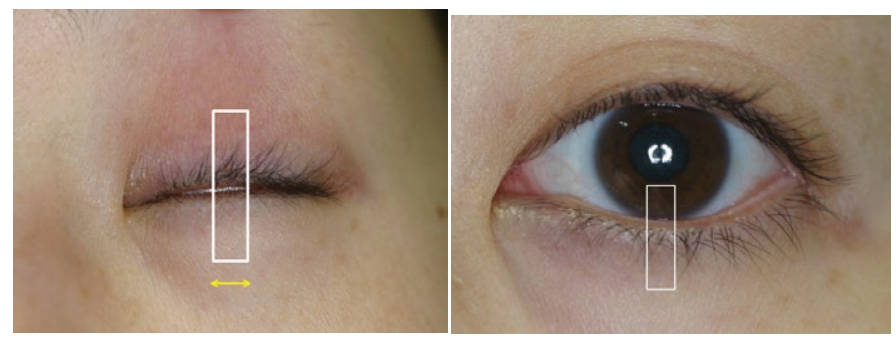

Figure 1. Density of eyelash (Upper eyelid: a) Lower eyelid: b) Scaled image with a digital camerawhereby the number of eyelashes growing in the central $2 \mathrm{~mm}$ of the upper and lower eyelids was recorded.

Correspondence to: Mamoru Kikuchi, Department of Plastic and Reconstructive Surgery, Saga University Hospital, Nabeshima 5-1-1, Sagacity, Saga, 849-8501, Japan, Tel: +81-952-34-2460; Fax: +81-952-34-2084; E-mail: Mamoru-osk@umin.ac.jp

Key words: eyelash, Japanese, length, density, angle

Received: January 26, 2015; Accepted: February 03, 2015; Published: February 07,2015 


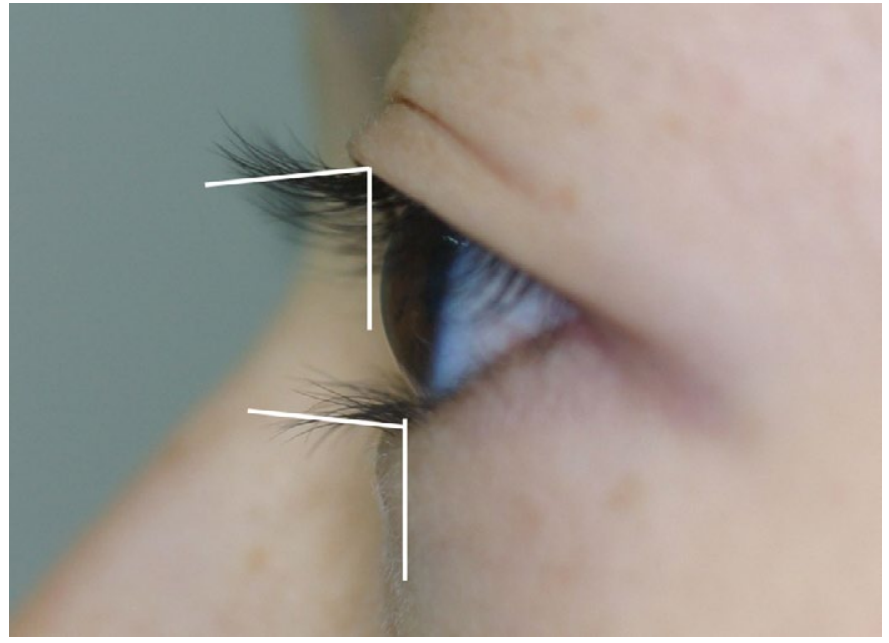

Figure 2. Lateral view of eyelash for measuring angles of an Asian female.

Table 1. Comparison of the length of male and female eyelashes.

\begin{tabular}{|l|l|c|c|c|}
\hline & & Male & Female & \\
\hline Parameters & & $(\mathrm{n}=25)$ & $(\mathrm{n}=25)$ & P-value \\
\hline Length $(\mathrm{mm})$ & Upper eyelid & $7.33 \pm 0.83$ & $7.47 \pm 0.68$ & $\mathrm{~N} / \mathrm{S}$ \\
\hline & Lower eyelid & $4.98 \pm 0.75$ & $5.56 \pm 0.60$ & $0.003^{* *}$ \\
\hline
\end{tabular}

Data are described as mean $\pm \mathrm{SD}$

${ }^{* *} \mathrm{P}<0.01$ (Mann-Whitney test)

$\mathrm{N} / \mathrm{S}$, not statistically significant

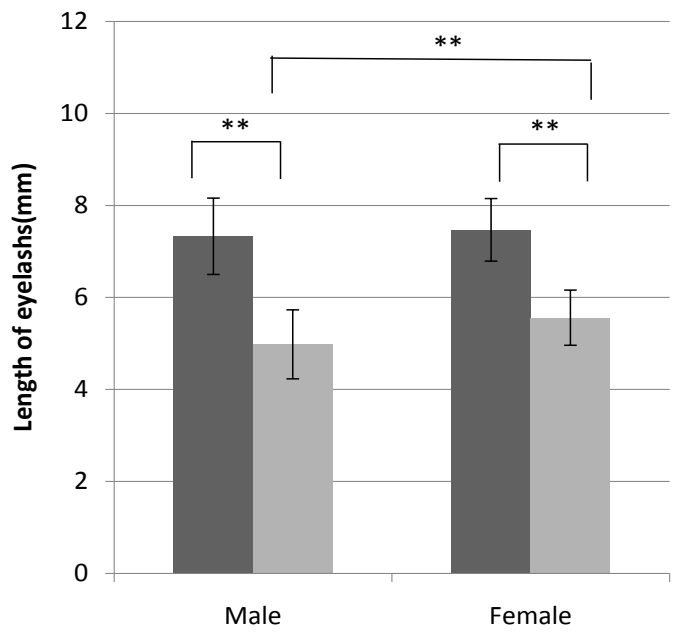

Upper eyelid

Lower eyelid

Figure 3. Comparison of the length of male and female eyelashes $(* * \mathrm{P}<0.01)$.

\section{Statistical analysis}

Statistical analysis was performed using $\mathrm{R}$ with significance set at $\mathrm{P}<0.05$. Mann-Whitney test was used to evaluate eyelash length and angle, and Student's t-test to evaluate eyelash density.

\section{Results}

Mean eyelash lengths in males and females were $7.33 \pm 0.83 \mathrm{~mm}$ and $7.47 \pm 0.68 \mathrm{~mm}$, respectively, for the upper eyelids, and $4.98 \pm 0.75 \mathrm{~mm}$ and $5.56 \pm 0.60 \mathrm{~mm}$, respectively, for the lower eyelids. No significant difference was found in the length of eyelashes on the upper eyelids by sex, but females had significantly longer eyelashes on the lower eyelids, and their upper eyelashes were significantly longer than their lower eyelashes (Table 1, Figure 3). Eyelash density showed a mean of 12.12 \pm 2.04 and $15.24 \pm 4.18$ for the upper eyelids, and $5.80 \pm 1.76$ and 6.24 \pm 1.16 for the lower eyelids, in males and females, respectively. In both sexes, the eyelashes on the upper eyelids were significantly thicker than those on the lower eyelids. No significant difference was found in the density of eyelashes on the lower eyelids by sex, but the eyelashes on the upper eyelids were significantly thicker in females than in males (Table 2, Figure 4).

With regard to the form of the eyelashes on the upper eyelids, 10 (40\%) were curly and $15(60 \%)$ were straight in males, whereas 19 (76\%) were curly and $6(24 \%)$ were straight in females. All lower eyelid eyelashes were curly (Table 3 ).

Mean angles of the eyelashes in males and females were $61.4^{\circ} \pm$ $13.7^{\circ}$ and $71.8^{\circ} \pm 13.5^{\circ}$, respectively, for the upper eyelids, and $90.0^{\circ}$ $\pm 10.1^{\circ}$ and $99.8^{\circ} \pm 10.8^{\circ}$, respectively, for the lower eyelids. Both upper and lower eyelids showed a significant difference by sex (Table 4, Figure 5).

Responses to the self-image questionnaire regarding eyelash length on the upper eyelids were as follows: short ( 2 males, 6 females), regular (20 males, 14 females), and long ( 3 males, 5 females); regarding eyelash thickness on the upper eyelids: thick ( 2 males, 4 females), normal ( 21 males, 19 females), and thin ( 2 males, 2 females). Among females, significant differences were found between the short and regular

Table 2. Comparison of the density of male and female eyelashes.

\begin{tabular}{|l|l|c|c|c|}
\hline & & Male & Female & \\
\hline Parameters & & $(\mathrm{n}=25)$ & $(\mathrm{n}=25)$ & P-value \\
\hline Density (number/2 mm) & Upper eyelid & $12.12 \pm 2.04$ & $15.24 \pm 4.18$ & $0.002^{* *}$ \\
\hline & Lower eyelid & $5.80 \pm 1.76$ & $6.24 \pm 1.16$ & $\mathrm{~N} / \mathrm{S}$ \\
\hline
\end{tabular}

Data are described as mean $\pm \mathrm{SD}$

${ }^{* *} \mathrm{P}<0.01$ (Student's t-test)

$\mathrm{N} / \mathrm{S}$, not statistically significant

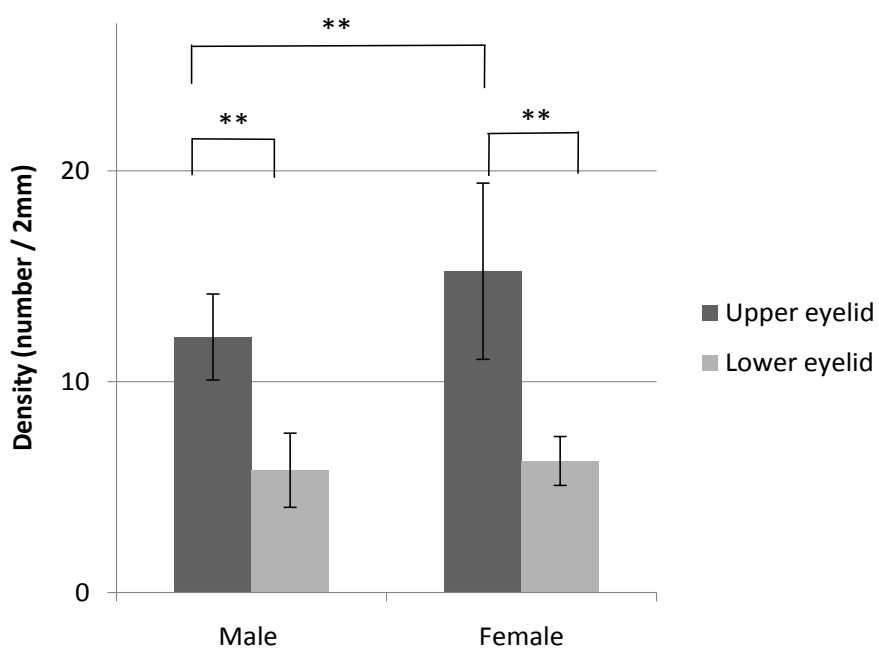

Figure 4. Comparison of the density of eyelashes $(* * \mathrm{P}<0.01)$.

Table 3: Form of the eyelashes.

\begin{tabular}{|l|l|c|c|c|}
\hline \multicolumn{2}{|c|}{} & Male & Female & Total \\
\cline { 3 - 5 } \multicolumn{2}{|c|}{} & $(\mathbf{n = 2 5}$ & $\mathbf{( n = 2 5 )}$ & $(\mathbf{n = 5 0 )}$ \\
\hline Upper eyelid & Curly & 10 & 19 & 29 \\
\hline & Straight & 15 & 6 & 21 \\
\hline Lower eyelid & Curly & 25 & 25 & 50 \\
\hline & Straight & 0 & 0 & 0 \\
\hline
\end{tabular}


Table 4: Comparison of the length of male and female eyelashes.

\begin{tabular}{|l|l|c|c|c|}
\hline & & Male & Female & \\
\hline Parameters & & $(\mathrm{n}=25)$ & $(\mathrm{n}=25)$ & P-value \\
\hline Angle $\left(^{\circ}\right)$ & Upper eyelid & $61.4 \pm 13.7$ & $71.8 \pm 13.5$ & $0.007^{* *}$ \\
\hline & Lower eyelid & $90.0 \pm 10.1$ & $99.8 \pm 10.8$ & $0.003^{* *}$ \\
\hline
\end{tabular}

Data are described as mean \pm SD

"P $<0.01$ (Mann-Whitney test)

$\mathrm{N} / \mathrm{S}$, not statistically significant

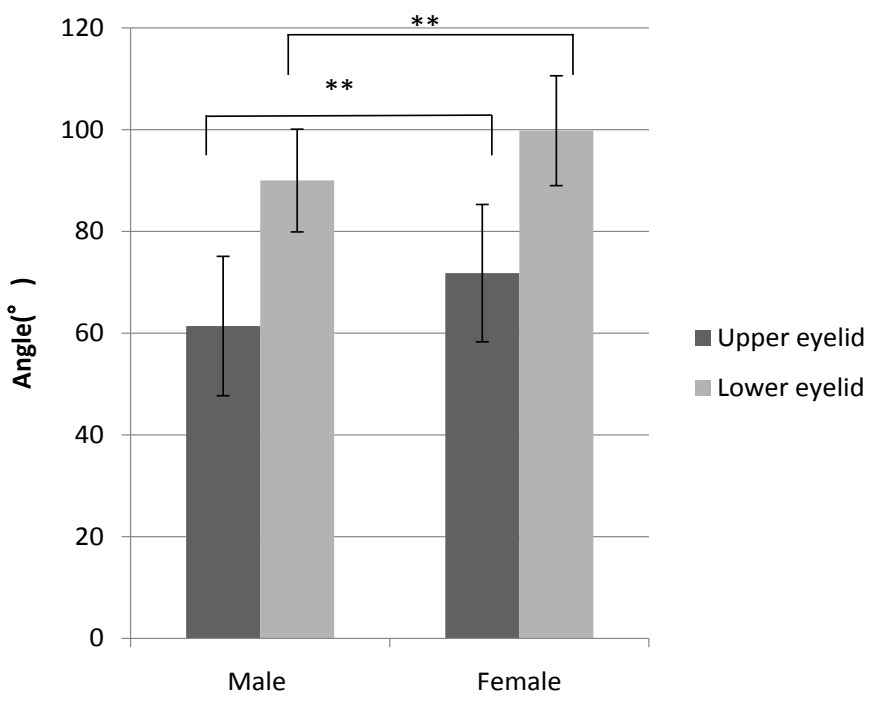

Figure 5. Comparison of the angle of eyelashes $(* * \mathrm{P}<0.01)$.

Table 5. Self-image on eyelashes.

\begin{tabular}{|l|c|c|c|c|c|c|}
\hline & \multicolumn{3}{|c|}{ Length } & \multicolumn{3}{c|}{ Density } \\
\hline & Short & Regular & Long & Thin & Normal & Thick \\
\hline Male & 2 & 20 & 3 & 2 & 21 & 2 \\
\hline Female & 6 & 14 & 5 & 4 & 19 & 2 \\
\hline
\end{tabular}

Table 6. Comparison of self-image on the length of upper eyelashes among females.

\begin{tabular}{|l|c|c|c|c|c|}
\hline Female & \multicolumn{4}{|c|}{ Self-image on eyelash length (upper eyelid) } \\
\hline & & \multicolumn{3}{|c|}{ Self-image } & \\
\hline Parameters & & Short $(\mathrm{n}=6)$ & Regular $(\mathrm{n}=14)$ & Long $(\mathrm{n}=5)$ & P-value \\
\hline Length $(\mathrm{mm})$ & Upper eyelid & $6.79 \pm 0.53$ & $7.67 \pm 0.66$ & $7.70 \pm 0.20$ & $0.01^{*}$ \\
\hline
\end{tabular}

Data are described as mean \pm SD

${ }^{*} \mathrm{P}<0.05$ (analysis of varaince)

groups, and between the short and long groups. Other items showed no significant difference (Tables 5 and 6, Figure 6).

\section{Discussion}

Eyelashes represent a facial feature that can greatly influence the overall impression ${ }^{5}$, and can be enhanced, especially in women, by mascara, eyeliner, or extensions. Various cosmetic products have been developed to extend or thicken eyelashes. Unfortunately, information on Asian eyelashes is limited. The present study obtained data from Asian (Japanese) individuals in their 20s and 30s, which is an age group with the highest interest in aesthetics.

We examined four items, i.e., length, density, form, and angle, which are considered morphologically important to characterize eyelashes. In both sexes, the eyelashes on the upper eyelids were significantly longer than those on the lower eyelids. Although no significant difference by

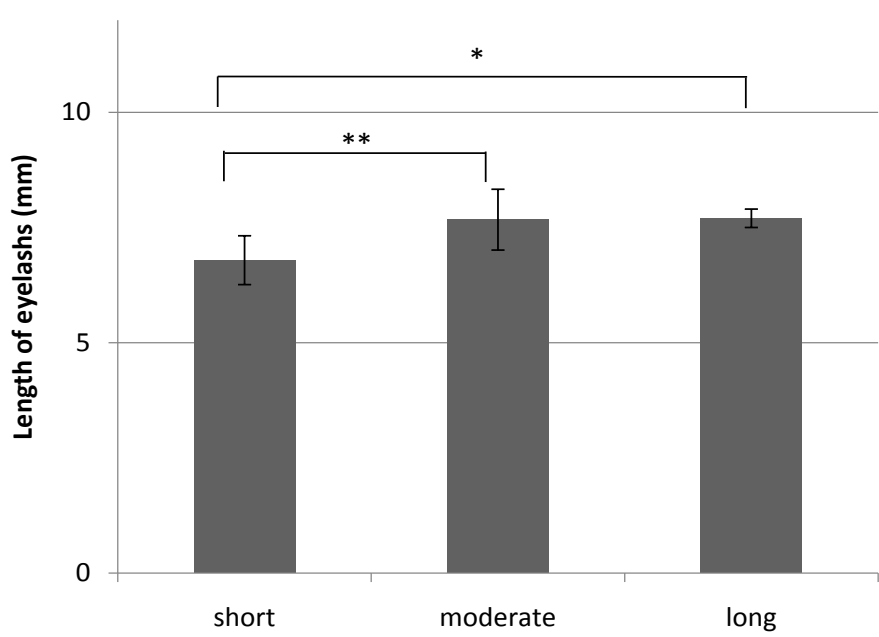

Figure 6. Self images of eyelashes.

sex was found in the length of eyelashes on the upper eyelids, females had significantly longer eyelashes on the lower eyelids. The length of the eyelashes on the upper eyelids represents the element most easily adjustable by makeup. In Japanese men and women, mean lengths at the middle of the eyelid were $7.33 \mathrm{~mm}$ and $7.47 \mathrm{~mm}$, respectively. The eyelashes on the upper eyelids were also significantly thicker than those on the lower eyelids in both males and females. The density of the eyelashes on the upper eyelids was significantly higher in females.

With respect to the form, both curly and straight eyelashes were observed on the upper eyelids, but the eyelashes on the lower eyelids were curly in all subjects. This is likely influenced by the fact that eyelashes on the lower eyelids grow in the upward direction against gravity. None of the subjects in this study had trichiasis.

A significant difference was found in eyelash angle between males and females. We requested the volunteers, especially females, not to use an eyelash curler or mascara on the day of the examination; however, the possible influence of daily makeup cannot be denied.

In the present study, all subjects participated in a self-image questionnaire survey regarding the length and thickness of their own eyelashes. Statistical analysis was not feasible due to large variations in the number of responses for each item. Yet, a significant difference was found in females across the three groups, only with regard to eyelash length.

Comparison of questionnaire results to our measurement values found that while individual evaluations did not differ significantly for length and density, the mean values for those who assessed their eyelashes as 'normal' was close to that of the overall mean. In other words, 'normal' eyelash length seems to be a standard and shared concept among Japanese people.

In conclusion, mean values obtained from the study above could be used as baseline values for eyelash reconstruction or treatment for eyelash hypotrichosis. Our study results pertaining to eyelash morphology represent one of a few assessments of eyelash characteristics in Japanese adults of both sexes. As such, these data provide basic information regarding eyelashes in Japanese individuals and could be utilized for aesthetic evaluations of eyelashes, eyelash hypotrichosis treatment, and treatment criteria for eyelash reconstruction. 


\section{References}

1. Franbourg A, Hallegot P, Baltenneck F, Toutain C, Leroy F (2003) Current research on ethnic hair. J Am Acad Dermatol 48: S115-S119. [Crossref]

2. Na JI, Kwon OS, Kim BJ, Park WS, Oh JK, et al. (2006) Ethnic characteristics of eyelashes: a comparative analysis in Asian and Caucasian females. Br J Dermatol 155: 1170-1176. [Crossref]
3. Harii K, Arase S, Tsuboi R, Weng E, Daniels S, et al. (2014) Bimatoprost for eyelash growth in Japanese subjects: two multicenter controlled studies. Aesthetic Plast Surg 38: 451-460. [Crossref]

4. Beer KR, Julius H, Dunn M, Wilson F (2013) Treatment of eyebrow hypotrichosis using bimatoprost: a randomized, double-blind, vehicle-controlled pilot study. Dermatol Surg 39: 1079-1087. [Crossref]

5. Thibaut S, De Becker E, Caisey L, Baras D, Karatas S, et al. (2010) Human eyelash characterization. Br J Dermatol 162: 304-310. [Crossref]

Copyright: (C2015 Mamoru Kikuchi. This is an open-access article distributed under the terms of the Creative Commons Attribution License, which permits unrestricted use, distribution, and reproduction in any medium, provided the original author and source are credited. 